\title{
Intracellular aggregated TRPVI is associated with lower survival in breast cancer patients
}

This article was published in the following Dove Press journal:

Breast Cancer - Targets and Therapy

\author{
Carlo Lozano ${ }^{1-3}$ \\ Claudio Córdova' \\ Ivanny Marchant ${ }^{1,2}$ \\ Rodrigo Zúñiga ${ }^{3}$ \\ Paola Ochova ${ }^{3}$ \\ Ricardo Ramírez-Barrantes ${ }^{4}$ \\ Wilfredo Alejandro \\ González-Arriagada ${ }^{2,5}$ \\ Belén Rodriguez' \\ Pablo Olivero ${ }^{1,2}$ \\ 'Laboratorio de Estructura y \\ Función Celular, Escuela de Medicina, \\ Facultad de Medicina, Universidad \\ de Valparaíso, Hontaneda, Valparaíso, \\ Chile; ${ }^{2}$ Centro Interoperativo en \\ Ciencias Odontológicas y Médicas, \\ Universidad de Valparaíso, Valparaíso, \\ Chile; ${ }^{3}$ Servicio de Anatomía \\ Patológica, Hospital Carlos van Buren, \\ San Ignacio, Valparaíso, Chile; ${ }^{4}$ Escuela \\ de Tecnologia Medica, Universidad \\ Andres Bello, Quillota, Viña del Mar \\ Chile; ${ }^{5}$ Patología y Diagnóstico Oral, \\ Facultad de Odontología, Universidad \\ de Valparaíso'Valparaíso, Chile
}

Correspondence: Carlo Lozano; Pablo Olivero

Laboratorio de Estructura y Función Celular, Escuela de Medicina, Facultad de Medicina, Universidad de Valparaíso, Hontaneda 2664, 2341386 Valparaíso, Chile

Email carlo.lburgos@gmail.com; pablo.olivero@uv.cl
Background: Breast cancer is a malignant disease that represents an important public health burden. The description of new molecular markers can be important to diagnosis, classification, and treatment. Transient receptor potential vanilloid 1 (TRPV1) polymodal channel is expressed in different neoplastic tissues and cell lines of breast cancer and associated with the regulation of tumor growth, tumor neurogenesis, cancer pain, and malignant progression of cancer. In primary and metastatic breast cancer tumors, TRPV1 is expressed during neoplastic transformation, invasive behavior, and resistance to cytotoxic therapy.

Objective: The objective of this study was to describe the subcellular distribution of TRPV1 in invasive breast carcinomas and its association with survival.

Methods: In 33 cases of invasive breast carcinomas, we identified immunohistochemical and immunofluorescent expression patterns of TRPV1 compared to healthy breast tissue. We characterized the expression of TRPV1 induced by estrogens in breast cancer cell lines MCF-7 and MDA to establish a model of the TRPV1-estrogen relationship regarding the malignant potential. We examined the association of TRPV1 patterns with patients' survival with the Kaplan-Meyer model, using the log-rank test at 5 years of follow-up. The relation of TRPV1 expression patterns to the St. Gallen breast cancer subtypes was also tested.

Results: Based on immunohistochemical expression pattern of TRPV1, we distinguished two main categories of breast cancer tissue, a "classical category" that exhibited diffuse expression of the channel and a "non-classical category" that expressed the channel in aggregates at the ER/Golgi and/or surrounding these structures. The classical pattern of TRPV1 was associated with a higher survival rate. In breast cancer cell lines, increasing doses of estrogens induced increased TRPV1 expression with nonclassical patterns at higher doses via a mechanism dependent on ER $\alpha$.

Conclusion: The expression and distribution of TRPV1 in invasive breast carcinomas may be considered as a biomarker for prognosis of the disease and a probable therapeutic target.

Keywords: immunohistochemistry, breast cancer, capsaicin receptors, vanilloid receptors, breast cancer prognosis

\section{Introduction}

As one of the most common cancers in women worldwide and despite the advances in cancer research and therapy, breast cancer remains a highly lethal malignant disease that represents a heavy burden to public health. The recommended treatment according to the molecular subtypes acknowledged by the St. Gallen consensus has shown favorable effects on patients' survival. ${ }^{1}$ However, the description of new associated molecules and the understanding of the mechanisms involved in the development 
of the disease will allow us to make a better prediction of the disease prognosis and possibly increase the accuracy in determining treatment subgroups based on novel targets able to improve the survival rates.

Advances have been made to propose treatment alternatives and specific drug targets for estrogen receptor (ER) alpha-positive, progesterone receptor (PR)-positive, and human epidermal growth factor receptor 2 (HER2)-positive breast cancers, whereas the most malignant triple-negative breast cancer phenotype, associated with higher rates of recurrence, metastasis, and worse prognosis, ${ }^{1}$ still has limited treatment choices.

Chronic inflammation has been revisited as an important risk factor associated with tumor progression. ${ }^{2}$ The progression of malignant tumors is reinforced by various molecules that are secreted during inflammation such as growth factors and cytokines that are common to immunological reactions and other inflammation phenomena. The molecules secreted by tumor cells contribute to develop their own microenvironment, modifying the extracellular matrix that surrounds them and the number of sensory neurons and modulating the immune system surveillance. ${ }^{3,4}$ These events protect the tumors of immune regulatory signals, favoring their growth and invasion, ${ }^{3}$ rendering the factors involved in these chronic inflammatory processes potential therapeutic targets for cancer.

The vanilloid family of transient receptor potential (TRP) channels consists of a broad variety of nonselective cation channels that integrate environmental and physicochemical signals for dynamic homeostatic control. This family of TRP channels may be activated by multiple paininducing stimuli including inflammatory conditions such as low $\mathrm{pH}(<6.0)$ and high temperature $\left(37^{\circ} \mathrm{C}-42^{\circ} \mathrm{C}\right)$ and chemicals such as capsaicin, anandamide, and inflammatory lipoxygenase metabolites. ${ }^{5}$ Additionally, the activation of TRPV family and other TRP channels has been involved in homeostasis of calcium, associated with inflammation, proliferation, angiogenesis, and regulation of cell death in tumors. ${ }^{6,7}$

Recent studies have reported the important role of TRP channels in an increasing number of diseases, TRPV1 being one of the most studied members of vanilloid family, which have been related to modulation of breast cancer cell death. ${ }^{8-10}$ The activation of TRPV1 in MCF-7 cells by its classical agonist capsaicin alone or in combination with other modulators such as MRS1477 and the chemotherapeutical agent cisplatin against breast cancer appears to contribute to induce cell death by apoptosis with mitochondrial mem- brane depolarization, production of ROS, and activation of caspases 3 and 9. ${ }^{11,12}$

Current evidence shows that low doses of capsaicin may induce apoptosis in tumor cells, while higher doses of capsaicin activate necrosis. This is dependent on the cell lineage and physiological context; for instance, in Hela cells with overexpression of TRPV1, nanomolar concentration of capsaicin induces a transitory calcium signal mediated by TRPV1 without any toxic effect, whereas micromolar concentration $(1-10 \mu \mathrm{M})$ of capsaicin induces apoptotic cell death and with doses as high as $100 \mu \mathrm{M}$ cell death follows a necrotic pattern. ${ }^{6}$

This special pattern of response of the channel to increasing doses of capsaicin may be very relevant in the context of advanced breast cancer tumors, where the overexpressing TRPV1 sensory neurons exhibit aberrant collateral axonal branching. ${ }^{4}$ The utilization of capsaicin in this context could reduce the aberrant activity of sensory neurons by blocking the tumor growth and, concomitantly, the pain sensation. ${ }^{4,10}$ Finding the minimum concentration necessary to induce cell death without causing excessive pain or inflammation can thus be considered as an important constraint to assess treatment efficacy of TRPV1 activators. ${ }^{10}$

Another clinically relevant characteristic of TRPV1 is their modulation by estrogens..$^{13,14}$ The available evidence suggest that $17 \beta$-estradiol is the only steroid that enhances the activity of TRPV1 channel. ${ }^{15,16}$ In addition, 17 $\beta$-estradiol exerts classical genomic effects increasing the expression of TRPV1 in uterine afferent neurons ${ }^{17}$ as well as in dorsal root ganglion cells via mechanisms dependent on ER $\alpha$ and ER $\beta$ with effects on the nociception mediated by primary afferent neurons. ${ }^{17,18}$

All these new evidence pointed out the importance of understanding the role of TRPV 1 in the progression of breast cancer and aroused enthusiasm among researchers on the way of a new potential target for treatment. However, until now, most of the evidence have emerged mainly from in vitro models, and the role of the channel in vivo remains to be elucidated. For the best of our knowledge, the possible effect of estrogens on the expression and function ofTRPV1 in human breast cancer has not been reported, although understanding this mechanism could be of special interest given the strict estrogen dependence of the malignancy of these tumor cells as indicated by St. Gallen Consensus. ${ }^{1}$

To explore a possible role of TRPV1 channel in human breast cancer, first, we determined the expression and patterns of subcellular distribution of TRPV1 in two breast cancer cell lines with different malignant potentials such as MCF-7 and 
MDA. Second, we verified the dose-effect of estrogens on the expression of TRPV1 in both cell lines and the dependence of this effect on ERs. Finally, we assessed the expression and localization patterns of TRPV1 in human breast carcinomas and their correlation with survival. We tested the association of the expression patterns of TRPV1 with the molecular subtypes of the St. Gallen Consensus. ${ }^{1}$

\section{Materials and methods Tissue samples and survival record}

A total of 33 paraffin-embedded tissue blocks, corresponding to mastectomies with invasive breast carcinomas, were collected from the Pathology Department at Carlos van Buren Hospital (January 1, 2010-June 30, 2012). The survival was determined at 5 years of follow-up, from medical records and the national register of death causes (one case was not considered in the analysis of survival due to lack of information about the cause of death). The study was approved by the Ethics Committee for Human Studies of the Carlos van Buren Hospital. All patients signed a written informed consent authorizing the use of their samples and the associated data in biomedical research purposes.

\section{Tissue microarray (TMA)}

Representative tumor areas were selected on H\&E-stained sections, and the slides were then overlaid on the original paraffin block. TMAs were constructed using a manual tissue arrayer (Sakura Co., Tokyo, Japan), with one representative cylindrical core of $3.0 \mathrm{~mm}$ diameter taken from each original tissue block and then arranged into a recipient of paraffin block. Samples of normal breast tissue were inserted in each recipient block as controls. A map specifying the exact position of each case was prepared to facilitate the interpretation of the immunohistochemical results.

\section{Immunohistochemistry and immunofluorescence}

Primary antibodies for TRPV1 (1:1,000; Abcam, Cambridge, MA, USA), ER (RTU; Dako), PR (RTU; Dako, Agilent, Santa Clara, CA, USA), HER2 (RTU; Dako), and Ki67 (RTU; Dako) were used. The tumor samples were fixed with $10 \%$ formalin for 12 hours before processing. The TMA was cut obtaining slices of $4 \mu \mathrm{m}$ thick. The immunohistochemical stain was performed with Dako Autostainer Link 48 using the EnVision FLEX system. Primary and secondary antibodies were incubated for 20 minutes. The EnVision FLEX chromogen DAB was incubated for 5 minutes. The slides were counterstained with Harris hematoxylin for 1 minute. We used mouse testis and cerebral vascular endothelium as controls for TRPV1 expression. To increase the robustness in detection of TRPV1, we used immunofluorescence techniques with two different antibodies directed at the terminal amino and carboxyl portions of the channel (C-TRPV1 and N-TRPV1, 1:1,000; Abcam) and Golgi-GFP imaging in MDA cells.

\section{Mapping of the subcellular distribution of TRPVI channel}

We utilized two fluorescent probes for detection of endoplasmic reticulum and Golgi complex and examined the colocalization with the channel signal applying an automated procedure (Figure 1B). The colocalization analysis identifies the spatial position where two or more digitalized stain signals converge. Finally, we generated four digital subcellular masks that identify the position of plasma membrane, cytoplasm, nucleus, and perinuclear region to easily distinguish the main cellular compartments where the channel could be distributed.

\section{Statistical analyses}

Statistical tests were performed using StatGraphics 5.1, 12.0 and SigmaPlot OriginPro 8.05 with ANOVA to determine statistical significance and multiple comparison procedure (Fisher's exact test for minimum statistical difference) with a confidence level of $95 \%$ to determine the pair of data that produces the difference. The statistical association of classical and nonclassical patterns of TRPV1 expression with the molecular St. Gallen subtypes LA, LB, HER2, and triple negative was examined with Fisher's exact test. We determined the association between categories of TRPV1 and survival with the Kaplan-Meyer model using the log-rank test at 5 years follow-up.

\section{Results}

The clinicopathological data of patients included in the study according to the categories of TRPV1 expression are described in Table 1.

\section{Estrogen-dependent expression and subcellular distribution of TRPVI in breast cancer cell lines}

Real-time PCR confirmed TRPV1 mRNA expression in both MCF-7 and MDA-MB-231 cell lines (Figure 1A). In addition, immunofluorescence detection confirmed the expression of TRPV1 protein in MCF-7 and also in MDA-MB-231 (Figure 1B). MCF-7 and MDA-MB-231 exhibited different subcellular distribution patterns. In the case of MCF-7, a high 
A

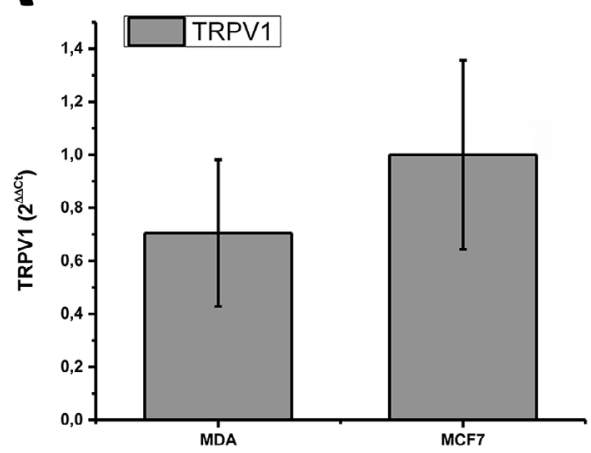

C

\begin{tabular}{ccc} 
Pearson R. Value & MCF7 & MDA \\
RETICULUM & 0,33 & 0,82 \\
GOLGI & 0,42 & 0,76 \\
NUCLEUS & 0,11 & 0,12 \\
MEMBRANE & 0,77 & 0,66 \\
CYTOPLASM & 0,79 & 0,44 \\
\hline
\end{tabular}
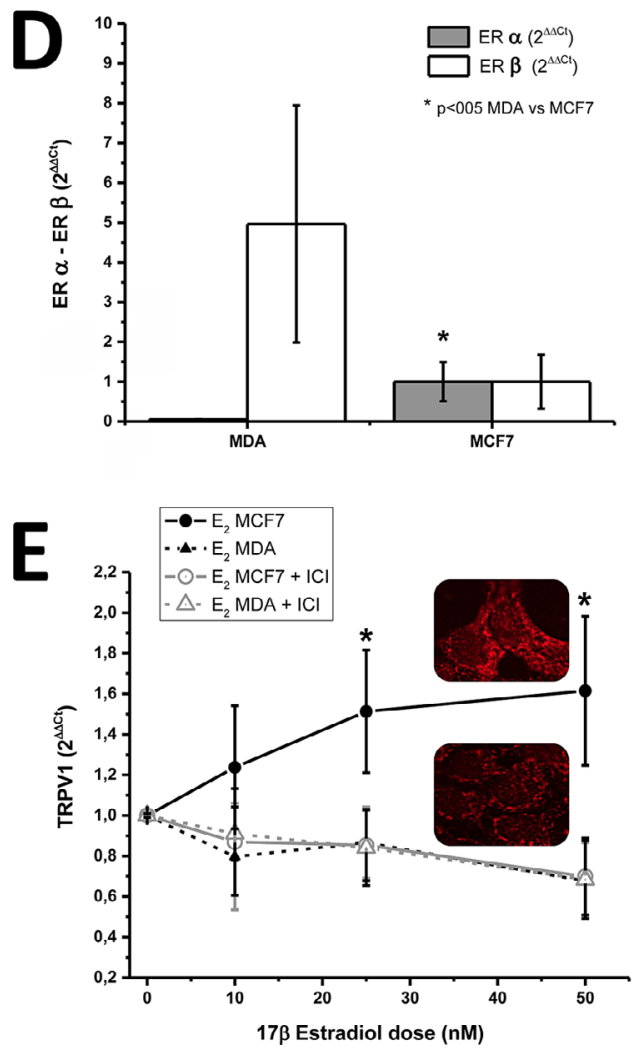

B

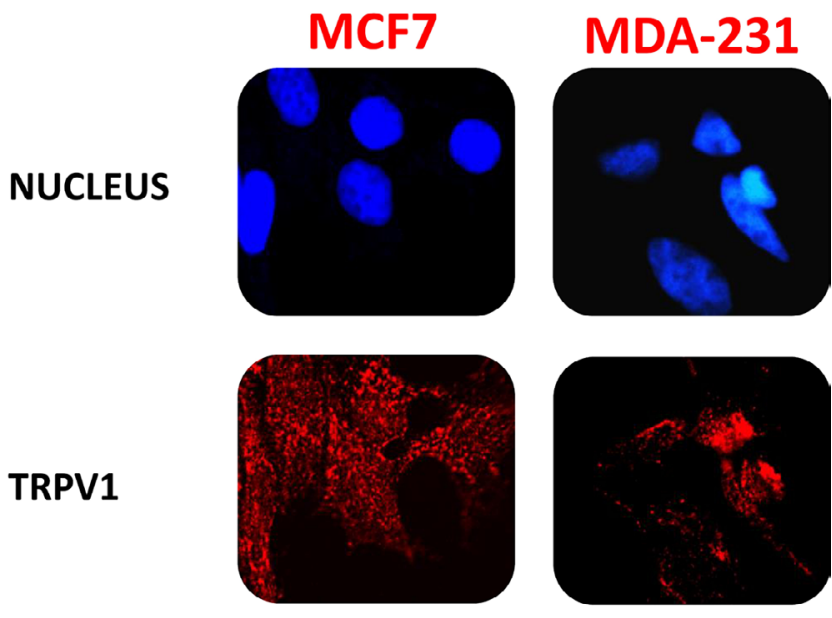

\author{
RETICULUM
}
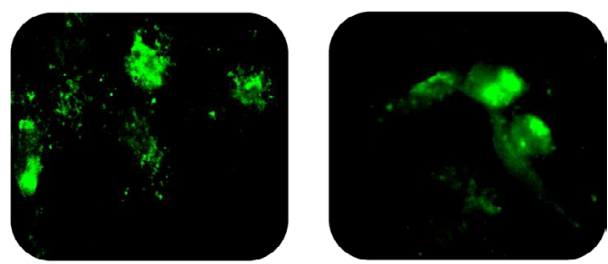

GOLGI
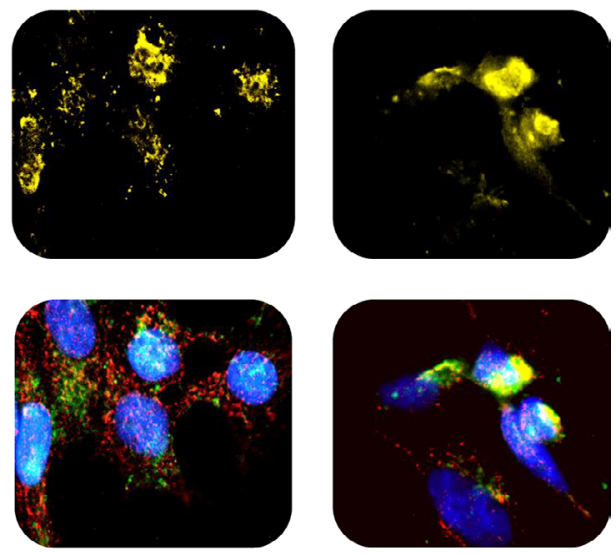

$\mathbf{F}$

\begin{tabular}{ccc}
\hline & MDA-MB-231 & MCF7 \\
ER & $\beta$ & $\alpha$ y $\beta$ \\
TRPV1 distribution & Non-Classical & Classical \\
Tumor phenotype & Her2 - TN & LA, LB \\
Malignancy & +++ & + \\
\hline
\end{tabular}

Figure I Transcription and subcellular distribution of TRPVI in MDA-MB-23I and MCF-7 cell lines.

Notes: (A) TRPVI mRNA expression in MDA-MB-23I and MCF-7 by qPCR did not reveal any statistical difference. (B) Different abundance and distribution of TRPVI in MDA-MB-23I and MCF-7. Image magnification: images was obtained in 100× (optical). After analysis process, digital magnification was I.25×. (C) Colocalization analysis results. In MDA-MB-23I, most of TRPVI protein followed the same subcellular distribution as the ER, whereas the classical pattern in MCF-7 did not colocalize with the ER/Golgi mark. (D) MDA-MB-23I and MCF-7 exhibited different expressions of ERs. In MDA-MB-23I, qPCR experiments showed expression of ER $\beta$ only, whereas MCF-7 expressed both ER $\alpha$ and ER $\beta(P<0.05)$. (E) The transcription of TRPVI in MCF-7 was induced by estradiol and blocked by the antagonist of ER ICI I82780. * $p>0.05$. $(\mathbf{F})$ In MCF-7, TRPVI did not present significant colocalization with any of the masks automatically generated, ie, plasma membrane, cytosol, and nucleus. This pattern can be classified as classical. On the other hand, MDA-MB-23I colocalizes more proteins in the cytosol mask, this pattern being classified as nonclassical.

Abbreviation: qPCR, quantitative PCR. 
Table I Clinical parameters according to TRPVI expression categories

\begin{tabular}{|l|l|l|l|l|}
\hline Parameters & Classical & Nonclassical & P-value & Total \\
\cline { 2 - 3 } & $\mathbf{n}(\%)$ & $\mathbf{n}(\%)$ & & \\
\hline T stage & & & & \\
TI/T2 & $18(62.0)$ & $11(37.9)$ & 0.614 & 29 \\
T3/T4 & $3(75)$ & $1(25)$ & & 4 \\
N stage & & & & \\
NI & $10(66.6)$ & $5(33.3)$ & $0.74 \mathrm{I}$ & 15 \\
N2-3 & $11(61.1)$ & $7(38.8)$ & & 18 \\
Clinical stage & & & & \\
I/II & $10(66.6)$ & $5(33.3)$ & $0.74 \mathrm{I}$ & 15 \\
III/IV & $11(6 I .1)$ & $7(38.8)$ & & 18 \\
St. Gallen & & & & \\
Lower malignancy & $19(73.1)$ & $7(26.9)$ & $0.029^{\mathrm{a}}$ & 26 \\
$\begin{array}{l}\text { (LA+LB) } \\
\text { Higher malignancy }\end{array}$ & $2(28.5)$ & $5(71.5)$ & & 7 \\
(HER2+TN) & & & & \\
Death & & & & \\
No & $17(73.9)$ & $6(26.0)$ & 0.060 & 23 \\
Yes & $4(40.0)$ & $6(60.0)$ & & 10 \\
Total & 21 & 12 & & 33 \\
\hline
\end{tabular}

Note: aSignificant association of the frequency of nonclassical pattern with higher malignancy St. Gallen subtypes.

Pearson coefficient value was obtained in membrane and cytoplasm, indicating that the expression of the channel was concentrated in these compartments, similar to the current channel distribution in several tissues normally expressing TRPV1 (Figure 1B and C). ${ }^{5}$ In the case of MDA-MB-231, the expression was predominant at the endoplasmic reticulum, Golgi, and perinuclear region (Figure 1B and C).

qPCR experiments to measure the level of mRNA of ERs did not show any difference in the amount of mRNA (Figure 1D) between MCF-7 and MDA-MB-231 cell lines. However, MDA-MB-231 and MCF-7 exhibited different expressions of ER subtypes. Although ER $\beta$ was present in both cell lines, only MCF-7 expressed ER $\alpha(P<0.05$; Figure 1D) indicating, as already suggested, that MDA-MB-231 is associated with a more malignant phenotype.

The response curve of TRPV1 at different doses of $17 \beta$-estradiol showed an increase in TRPV1 mRNA expression only in MCF-7 cells (Figure 1E). Notably, this effect was completely reverted by the inhibitor of ERs $\alpha$ and $\beta$ ICI 182780 , suggesting that the channel expression was due to classical 17ß-estradiol genomic effect that was dependent on ER $\alpha$. These results are summarized in Figure 1E, indicating that TRPV1 is expressed in both cell lines but the estrogeninduced expression pattern of TRPV1 changes, probably depending on the malignancy of the tumor. Figure 1F summarizes the parameters that describe the cellular model of breast cancer according to the degree of malignancy, adding the expression pattern of TRPV1.

\section{TRPVI expression and subcellular distribution in human breast cancer samples}

The immunofluorescence of C-TRPV1 and N-TRPV1 antibodies matched with the immunohistochemical results, confirming the positive and negative results of TRPV1 expression. Immunofluorescence was unable to detect a welldefined expression pattern of the channel, but it was useful to demonstrate that the perinuclear expression corresponds to the presence of the channel at the ER/Golgi complex (Figure 1A). The immunohistochemical assays revealed distinctive patterns of subcellular localization of TRPV1 in breast cancer samples. Among the specimens expressing TRPV1, we identified the following: 1) An intracellular diffuse pattern that was similar to the pattern of TRPV1 expression found in control tissues, as the epithelial cells in healthy breast (acini and ducts), mice seminiferous tubules, and capillary endothelium in brain tissue (data not shown). This pattern corresponded to the classical category. 2) A pattern in aggregates at the ER/Golgi. 3) A group that, in addition to TRPV1 aggregates at the ER/Golgi, exhibited a relatively diffuse distribution of TRPV1 in the cytosol. These two last forms of presentation were grouped as the "nonclassical" category (Figure 2A and B). TRPV1 expression was undetectable in two specimens, which were included in the classical category. In all, 21 cases were classified as classical and 12 cases were classified as nonclassical category (Figure 2C). The classical pattern of TRPV1 was mainly observed in low-grade St. Gallen groups, while the nonclassical pattern of TRPV1 was observed in more aggressive St. Gallen groups $(P<0.05$; Table 1 and Figure 2C). The Kaplan-Meyer survival curves indicate better prognosis in patients with the classical pattern of immunohistochemical expression of TRPV1 (log-rank test $<0.05$; Figure 2D).

\section{Discussion}

Currently located at the plasma membrane, TRPV1 nonselective cation channel is especially permeable to $\mathrm{Na}^{+}$and $\mathrm{Ca}^{2+}$. Various stimuli such as capsaicin, heat, acid pH, endogenous lipids, and some inflammatory mediators may activate this channel, which is considered a permanent and fast controller of the cellular homeostasis. ${ }^{19,20}$ In the nervous system, TRPV1 is constitutively expressed mainly in neurons linked to the routes of transmission of pain, although bronchial epithelium, gastrointestinal tract, nasal epithelial cells, and endothelial 
A

\section{Breast Cancer}
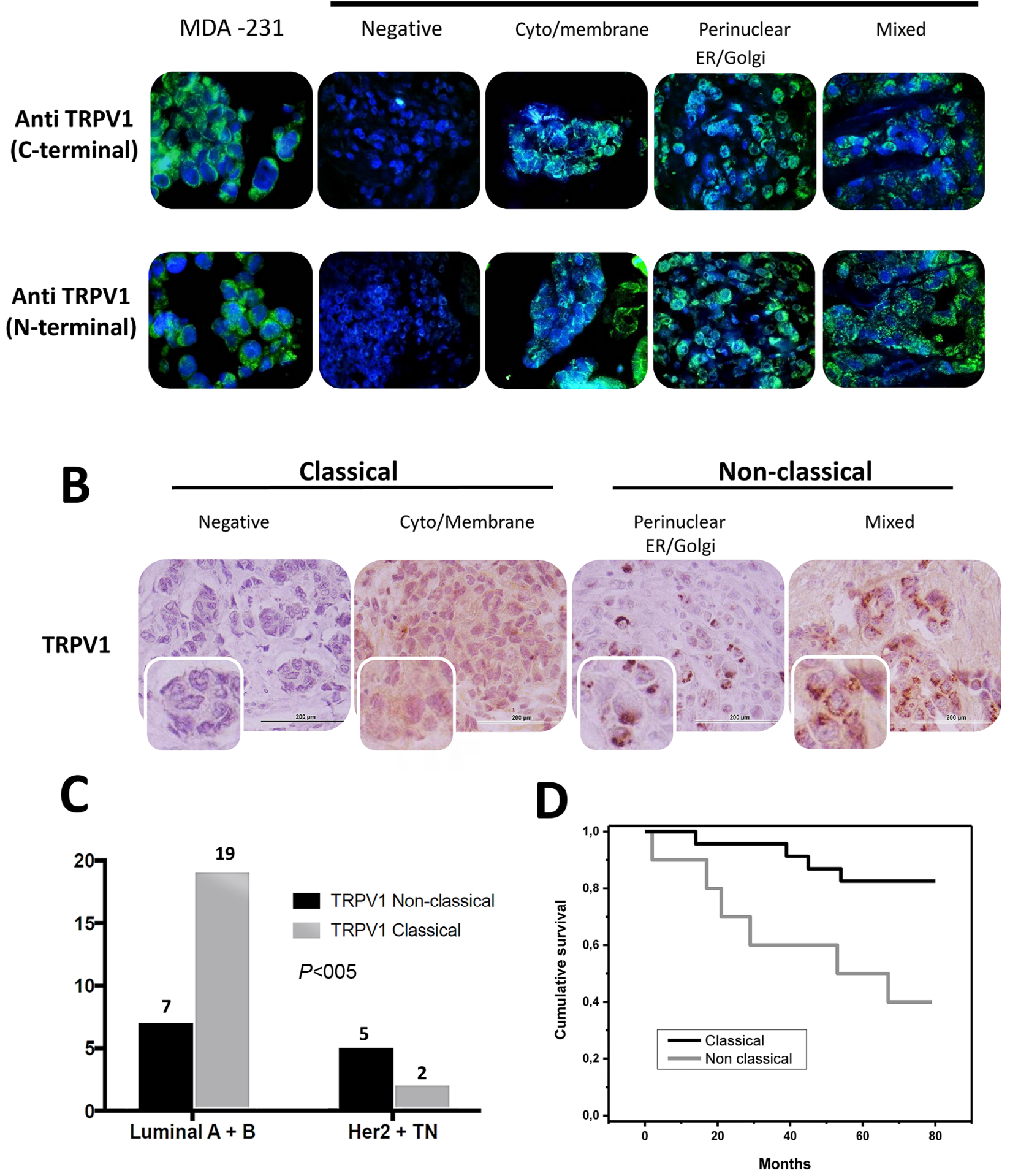

\begin{tabular}{|c|c|c|c|c|c|c|c|c|c|}
\hline & Her2 & ER & $\overline{P R}$ & Ki67 & & Her2 & ER & $\overline{P R}$ & Ki67 \\
\hline LA & - & + & + & $<14 \%$ & H & + & - & - & \\
\hline LB & - & + & + & $>14 \%$ & TN & - & - & - & \\
\hline
\end{tabular}

Comparison of survival curves (Log-rank test)

Significance $P=0.012$

Figure 2 Nonclassical pattern of TRPVI identifies higher malignancy breast carcinomas.

Notes: (A) The immunofluorescence of C-TRPVI and N-TRPVI antibodies confirmed the expression of TRPVI in breast cancer, with a diffuse expression pattern. (B) The immunohistochemical detection of TRPVI shows a classical pattern at the plasma membrane and cytosol, and a nonclassical pattern with aggregates of TRPVI at the ER/Golgi and/or a relatively diffuse distribution of the channel at the surrounding cytosol. (C) The classical TRPVI pattern was more frequent in lower malignancy St. Gallen subtypes luminal A + luminal B compared to the most aggressive subtypes Her 2 + triple negative. (D) Survival curves according to the pattern of TRPVI subcellular distribution. 
cells have also been shown to express the channel, ${ }^{21,22}$ in particular, in the context of inflammatory responses. Constitutive expression in nociceptive neurons follows a classical pattern of TRPV1 localization at the plasma membrane strengthening its role as a pain modulator. Differently, the inducible expression exhibits a special subcellular distribution that has not yet been fully described. It is expected that a better understanding of this inducible behavior of TRPV1 will provide some explanations on the homeostatic mechanisms developed by tumors achieving higher malignancy related to TRPV1 strategies.

The TRP channels are highly expressed in different types of cancers, ${ }^{23}$ and some authors have suggested its role as a therapeutic target in breast cancer. ${ }^{24}$ In tumor cells, TRP channels may contribute to cancer progression through changing the activity of signaling pathways associated with variation in intracellular $\mathrm{Ca} 2+$ levels, particularly by modifying the cell proliferation and migration. ${ }^{25,26}$ The transcriptional regulation of TRP channels under hormonal control is the main studied mechanism in hormone-dependent cancers, such as prostate and breast cancers. ${ }^{7}$

In the present study, individuals with nonclassical patterns of TRPV1 expression showed lower survival rates than the individuals with classical patterns. In addition, TRPV1 nonclassical aggregates were associated with the more aggressive St. Gallen subtypes, suggesting that breast cancer malignancy and progression could be related to altered turnover of the channel, possibly due to postransductional modifications. Further explorations are needed to elucidate whether these aggregates are causal or rather a consequence of the tumor behavior, though this phenomenon seems to reflect changes in important cell functions that depend on the availability of TRP channels at the plasma membrane. Indeed, dynamic changes in the availability of TRP channels at the plasma membrane have been put forward as potentially important regulatory mechanisms of TRP channel function in vivo. ${ }^{27}$ Accumulation of the channel in Golgi complex may have functional implications. The intracellular sequestration of TRPV1 in neurons has been associated with the predominance of the monomeric form of channel, instead of the tetrameric form, ${ }^{28}$ which may result in accumulates of nonfunctional forms of the channel. We interpret the lack of expression of the channel in the nonclassical category as due to the "abduction" of TRPV1 by the endomembrane system, as an intent to optimize cellular performances in stress conditions imposed by the tumor environment.

Estrogens have been demonstrated to exert transcriptional regulation of TRPV $6,{ }^{7}$ in addition to their implication in modulating nociception through TRPV1 receptor. $^{29}$ The immunohistochemical expression of TRPV1 in biopsies of breast cancer has not been reported before, but the overexpression of the channel in cell lines of this type of cancer has been demonstrated, ${ }^{9,10,30}$ similar to another channel of the same family, TRPV6, which is modified by estradiol/ progesterone and associated with proliferation of neoplastic cells. ${ }^{31}$

Our results indicate a dose-dependent relationship between the expression of TRPV1 and estrogens in cells that express ER $\alpha$. In addition to the abundance of TRPV1, increasing doses of $17 \beta$-estradiol induced an increased concentration of the channel surrounding the nucleus, in relation to the ER/Golgi system, which might reflect strategies that favor the survival of tumor cells and may predict their progression to more malignant states. Therefore, it is likely that estrogen-dependent perinuclear accumulation of TRPV1 in breast cancer tumors can indicate some stationary state that confers comfortable survival conditions to the tumor and worsened prognosis to host individuals.

These observations put the focus on modulators of TRPV1 channels turnover as potential pharmacological targets in cancer subtypes and/or tumors expressing mainly nonclassical patterns of TRPV1. Nevertheless, until the nonclassical pattern is better characterized, the conductive form of TRPV1 in classical membrane localization offers a possible target that merits clinical exploration: sensitizing tumors with agonists as capsaicin may have favorable therapeutic effects on breast cancer, via the regulation of calcium homeostasis and mitochondrial activity, ${ }^{8,10,23,32}$ thus controlling the proliferation, apoptosis, and migration ${ }^{9,33,34}$ of tumor cells. As an optimistic perspective, we hope to extend our analytic strategies for anticipating the tumor behavior focusing on understanding the role of TRPV1 subcellular distribution during the cancer progression.

\section{Conclusion}

The intracellular TRPV1 aggregates in breast carcinoma are associated with particular biological behavior. Of clinical relevance, the expression of the nonclassical pattern is associated with worse prognosis and lower survival, suggesting that TRPV1 could be a therapeutic target for the most aggressive St. Gallen breast cancer subtypes. If confirmed in larger samples, the strength of our findings could introduce new criteria in the classification of breast cancer patients and inspire personalized therapeutic approaches based on homeostatic attack of tumor diseases. 


\section{Disclosure}

The authors report no conflicts of interest in this work.

\section{References}

1. Harbeck N, Thomssen C, Gnant M. St. Gallen 2013: brief preliminary summary of the consensus discussion. Breast Care (Basel). 2013;8(2):102-109.

2. Schäfer M, Werner S. Cancer as an overhealing wound: an old hypothesis revisited. Nat Rev Mol Cell Biol. 2008;9(8):628-638.

3. Grivennikov SI, Greten FR, Karin M. Immunity, inflammation, and cancer. Cell. 2010;140(6):883-899.

4. Austin M, Elliott L, Nicolaou N, Grabowska A, Hulse RP. Breast cancer induced nociceptor aberrant growth and collateral sensory axonal branching. Oncotarget. 2017;8(44):76606-76621.

5. Ramírez-Barrantes R, Cordova C, Poblete H, et al. Perspectives of TRPV1 Function on the Neurogenesis and Neural Plasticity. Neural Plast. 2016;2016:1-12.

6. Ramírez-Barrantes R, Córdova C, Gatica S, et al. Transient Receptor Potential Vanilloid 1 Expression Mediates Capsaicin-Induced Cell Death. Front Physiol. 2018;9:1-10.

7. Gkika D, Prevarskaya N. Molecular mechanisms of TRP regulation in tumor growth and metastasis. Biochim Biophys Acta. 2009;1793:953-958.

8. Koşar PA, Nazıroğlu M, Övey İS, Çiğ B. Synergic Effects of Doxorubicin and Melatonin on Apoptosis and Mitochondrial Oxidative Stress in MCF-7 Breast Cancer Cells: Involvement of TRPV1 Channels. $J$ Membr Biol. 2016;249(1-2):129-140.

9. Wu TT, Peters AA, Tan PT, Roberts-Thomson SJ, Monteith GR. Consequences of activating the calcium-permeable ion channel TRPV1 in breast cancer cells with regulated TRPV1 expression. Cell Calcium. 2014;56(2):59-67.

10. Pecze L, Jósvay K, Blum W, et al. Activation of endogenous TRPV1 fails to induce overstimulation-based cytotoxicity in breast and prostate cancer cells but not in pain-sensing neurons. Biochim Biophys Acta. 2016;1863(8):2054-2064.

11. Nazıroğlu M, Çiğ B, Blum W, et al. Targeting breast cancer cells by MRS1477, a positive allosteric modulator of TRPV1 channels. PLoS One. 2017;12(6):1-19.

12. Nur G, Nazıroğlu M, Deveci HA. Synergic prooxidant, apoptotic and TRPV1 channel activator effects of alpha-lipoic acid and cisplatin in MCF-7 breast cancer cells. J Recept Signal Transduct Res. 2017;37(6):569-577.

13. Irnaten M, Blanchard-Gutton N, Harvey BJ. Rapid effects of 17betaestradiol on epithelial TRPV6 Ca2+ channel in human T84 colonic cells. Cell Calcium. 2008;44(5):441-452.

14. Irnaten M, Blanchard-Gutton N, Praetorius J, Harvey BJ. Rapid effects of 17beta-estradiol on TRPV5 epithelial Ca2+ channels in rat renal cells. Steroids. 2009;74(8):642-649.

15. Chen SC, Chang TJ, Wu FS. Competitive inhibition of the capsaicin receptor-mediated current by dehydroepiandrosterone in rat dorsal root ganglion neurons. JPharmacol Exp Ther. 2004;311(2):529-536.

16. Lu YC, Chen CW, Wang SY, Wu FS. 17Beta-estradiol mediates the sex difference in capsaicin-induced nociception in rats. J Pharmacol Exp Ther. 2009;331(3):1104-1110.

Breast Cancer - Targets and Therapy

\section{Publish your work in this journal}

Breast Cancer - Targets and Therapy is an international, peerreviewed open access journal focusing on breast cancer research, identification of therapeutic targets and the optimal use of preventative and integrated treatment interventions to achieve improved outcomes, enhanced survival and quality of life for the cancer patient.
17. Cho T, Chaban VV. Interaction between $\mathrm{P} 2 \mathrm{X} 3$ and oestrogen receptor (ER)alpha/ERbeta in ATP-mediated calcium signalling in mice sensory neurones. J Neuroendocrinol. 2012;24:789-797.

18. Cho T, Chaban VV. Expression of $\mathrm{P} 2 \mathrm{X} 3$ and TRPV1 receptors in primary sensory neurons from estrogen receptors- $\alpha$ and estrogen receptor- $\beta$ knockout mice. Neuroreport. 2012;23(9):530-534.

19. O'Neill J, Brock C, Olesen AE, Andresen T, Nilsson M, Dickenson AH. Unravelling the mystery of capsaicin: a tool to understand and treat pain. Pharmacol Rev. 2012;64(4):939-971.

20. Mohapatra DP, Nau C. Desensitization of capsaicin-activated currents in the vanilloid receptor TRPV1 is decreased by the cyclic AMP-dependent protein kinase pathway. J Biol Chem. 2003;278(50):50080-50090.

21. Li S, Bode AM, Zhu F, et al. TRPV1-antagonist AMG9810 promotes mouse skin tumorigenesis through EGFR/Akt signaling. Carcinogenesis. 2011;32(5):779-785.

22. Seki N, Shirasaki H, Kikuchi M, Sakamoto T, Watanabe N, Himi T. Expression and localization of TRPV1 in human nasal mucosa. Rhinology. 2006;44(2):128-134.

23. Prevarskaya N, Zhang L, Barritt G. TRP channels in cancer. Biochim Biophys Acta. 2007;1772(8):937-946.

24. Ouadid-Ahidouch H, Dhennin-Duthille I, Gautier M, Sevestre H, Ahidouch A. TRP channels: diagnostic markers and therapeutic targets for breast cancer? Trends Mol Med. 2013;19(2):117-124.

25. Mergler S, Derckx R, Reinach PS, et al. Calcium regulation by temperature-sensitive transient receptor potential channels in human uveal melanoma cells. Cell Signal. 2014;26(1):56-69.

26. Monteith GR, Davis FM, Roberts-Thomson SJ. Calcium channels and pumps in cancer: changes and consequences. $J$ Biol Chem. 2012;287(38):31666-31673.

27. Ghosh D, Segal A, Voets T. Distinct modes of perimembrane TRP channel turnover revealed by TIR-FRAP. Sci Rep. 2014;4:7111.

28. Liedtke WB, Heller S. TRP Ion Channel Function in Sensory Transduction and Cellular Signaling Cascades. TRP Ion Channel Function in Sensory Transduction and Cellular Signaling Cascades. Boca Raton, Forida, USA: CRC Press/Taylor \& Francis; 2007.

29. Cho T, Chaban VV. Interaction between $\mathrm{P} 2 \mathrm{X} 3$ and oestrogen receptor $(\mathrm{ER}) \alpha / \mathrm{ER} \beta$ in ATP-mediated calcium signalling in mice sensory neurones. J Neuroendocrinol. 2012;24(5):789-797.

30. Weber LV Al-Refae K, Wölk G, et al. Expression and functionality of TRPV1 in breast cancer cells. Breast cancer (Dove Med Press). 2016;8:243-252.

31. Bolanz KA, Hediger MA, Landowski CP. The role of TRPV6 in breast carcinogenesis. Mol Cancer Ther. 2008;7(2):271-279.

32. Shapovalov G, Ritaine A, Skryma R, Prevarskaya N. Role of TRP ion channels in cancer and tumorigenesis. Semin Immunopathol. 2016;38(3):357-369.

33. Vercelli C, Barbero R, Cuniberti B, et al. Transient receptor potential vanilloid 1 expression and functionality in mcf-7 cells: a preliminary investigation. J Breast Cancer. 2014;17(4):332-338.

34. Ziglioli F, Frattini A, Maestroni U, Dinale F, Ciufifeda M, Cortellini P. Vanilloid-mediated apoptosis in prostate cancer cells through a TRPV-1 dependent and a TRPV-1-independent mechanism. Acta Biomed. 2009;80(1):13-20.
The manuscript management system is completely online and includes a very quick and fair peer-review system, which is all easy to use. Visit http://www.dovepress.com/testimonials.php to read real quotes from published authors. 